\title{
Upregulation of PNCK Promotes Metastasis and Mediates Angiogenesis Via NF-KB/VEGF Signaling Pathway in Nasopharyngeal Carcinoma
}

\section{Xiaochuan Chen}

Fujian Provincial Cancer Hospital

\section{Youliang Weng}

Fujian Provincial Cancer Hospital

\section{Ying Li}

Fujian Provincial Cancer Hospital

Xiandong Lin

Fujian Provincial Cancer Hospital

\section{Zongwei Huang}

Fujian Provincial Cancer Hospital

\section{Yuhui Pan}

Fujian Provincial Cancer Hospital

\section{Wenquan Hong}

Fujian Provincial Cancer Hospital

\section{Wankai Fu}

Shanghai Cancer Hospital: Fudan University Shanghai Cancer Center

Wanzun Lin

Shanghai Cancer Hospital: Fudan University Shanghai Cancer Center

Sufang Qiu ( $\sim$ sufangqiu@fjmu.edu.cn )

Department of Radiation Oncology,Fujian Medical University Cancer Hospital,Fujian Cancer Hospital https://orcid.org/0000-0001-7660-6955

\section{Primary research}

Keywords: nasopharyngeal carcinoma, PNCK, metastasis, angiogenesis, zebrafish

Posted Date: May 6th, 2021

DOl: https://doi.org/10.21203/rs.3.rs-493211/v1

License: (c) (1) This work is licensed under a Creative Commons Attribution 4.0 International License. Read Full License 


\section{Abstract}

Background: Previous studies suggest that pregnancy upregulated non-ubiquitous calmodulin kinase (PNCK) depletion represses proliferation and promotes apoptosis of nasopharyngeal carcinoma (NPC) cells. However, the underlying mechanism of the regulatory role of PNCK on NPC invasion and metastasis remains unclear.

Methods: The PNCK expression level was detected in specimens of non-metastatic and metastatic NPC by mRNA sequencing and immunohistochemistry. The wound-healing and transwell assays were employed to explore the ability of PNCK-mediated cell migration and invasion. Xenograft tumour model was adopted for monitoring tumour progression and lung metastasis was assessed by tail vein injection. Zebrafish model was used to explore the potential action of PNCK on tumor angiogenesis. We used gene set enrichment analysis and correlation analysis for mechanical investigation.

Results: Human metastatic NPC samples showed increased PNCK expression in both mRNA and protein levels. In vitro assay showed that shRNA mediated silencing of PNCK inhibited NPC cell migration and invasion, while PNCK overexpression enhanced the metastatic potential of NPC cells. Additionally, in vivo experiments revealed that overexpression of PNCK favored the metastatic foci formation in the lung and tumor angiogenesis. Consistently, a significant increase of metastatic nodules in lungs in mice and the vascular-labeled fluorescence signal of zebrafish inoculated with PNCK-overexpression cells compared to control group. Pathway analysis identified that the depletion of PNCK may inhibits NF-KB/VEGF signaling pathway in NPC.

Conclusions: These findings reveal that PNCK knockdown could suppress tumor metastasis and angiogenesis by regulating NF-KB/VEGF signaling pathway in NPC, suggesting that PNCK may represent a therapeutic target for NPC individualized treatment.

\section{Background}

Nasopharyngeal carcinoma (NPC) is a common malignancy originating in the epithelium of nasopharynx with unique geographical distribution and high incidence in Southeast Asia and Southern China [1, 2]. Since the deep anatomic position and concealed early symptoms, $80 \%-90 \%$ of NPC patients are first diagnosed as the middle- and advanced-stages disease [3,4]. Moreover, cervical lymph node metastases and aggressiveness of adjacent tissues have already occurred in almost $75 \%-90 \%$ of patients when first diagnosed due to the feature of high invasion and early metastasis [5]. Despite the great progress made for treatment of NPC, such as radiotherapy, which as the main treatment strategy, combined chemotherapy, targeted therapy, or immunotherapy, invasion and metastasis remains the key point impacting prognosis of patients [6]. Thus, is vital to further explore the molecular mechanisms of underlying invasion and metastasis which may contribute to develop more effective and novel options for individualized therapy for NPC. 
Pregnancy up-regulated nonubiquitous calmodulin (CaM) kinase (PNCK) as a unique member of CaM kinases I family, impacts various biological functions, involving the important roles in human malignancies [7-10]. Some previous researches have focus on the role of PNCK in carcinogenesis. It has been confirmed that PNCK is upregulated in breast cancer, renal carcinoma and NPC [11-13]. It has been reported that PNCK is related with increased proliferation properties of HER-2-positive breast cancer cells [14]. Furthermore, it demonstrated that PNCK plays a vital role in regulating cytoplasmic and nuclear signal transduction, thus possessing diverse biological functions over recent years [15]. Our previously study found that knockdown of PNCK could suppress tumor cell proliferation and promote apoptosis of NPC [11]. However, the underlying mechanism and functions of PNCK which exerts its role in invasion and metastasis of NPC remains unclear.

Here, we detected PNCK expression in NPC metastatic and non-metastatic tissue and revealed a significant high expression metastatic tissue. The results showed that upregulated PNCK significantly promoted the metastatic ability of NPC cells by in vitro and vivo studies. Furthermore, we demonstrated that PNCK mediated NPC cells metastasis by the NF-KB pathway and PNCK promoted NPC angiogenesis. Taken together, our study clarifies a potential mechanism that PNCK overexpression promotes NPC cells metastasis and identifies a prognostic biomarker and target for NPC individualized treatment.

\section{Methods}

\section{Patients and clinical specimens}

Totally 5 freshly frozen metastatic and 5 non-metastatic NPC tissue were collected in Fujian Cancer Hospital by biopsy between January 2015 and March 2015 in this study. Gene expression analysis was performed using 132 paraffin-embedded NPC specimens obtained from patients diagnosed with primary NPC without any relevant treatments before biopsy. Two senior pathologists confirmed the pathology who were blind to patients' clinical information. Protocols for sample collection was performed with approval of the ethics committee of Fujian Medical University Cancer Hospital, Fujian Cancer Hospital. All patients had signed written informed consent.

For an independent NPC dataset (GSE102349 from GPL11154 platform) from the Gene Expression Omnibus (GEO) (https://www.ncbi.nlm.nih.gov/gds/), 113 patients were included according with the following selection criteria: (a) definite histologically diagnosis of NPC; (b) available RNA-seq data.

\section{Cell culture and cell transfection}

Two human NPC cell lines (CNE-2 and SUNE-1) were obtained from Shanghai Institutes for Biological Sciences. All cells were cultured in RPMI 1640 medium containing 10\% FBS, $100 \mathrm{U} / \mathrm{mL}$ penicillin, and 100 $\mathrm{U} / \mathrm{mL}$ streptomycin, maintained at $37^{\circ} \mathrm{C}$ in $5 \% \mathrm{CO}_{2}$ incubator. To generate PNCK overexpression and knockout stable transfectants, we introduced lentiviral vectors expressing GFP or control vector in CNE-2 and SUNE-1 cells. It was commercially performed to construct plasmid and package lentivirus (Genechem Co. Ltd., Shanghai, China). 


\section{RNA-seq: data analysis}

The freshly frozen tissue and CNE-2 cell were useful for mRNA sequencing. Total RNA was extracted with Trizol (Invitrogen) and assessed with Agilent 2100 BioAnalyzer (Agilent Technologies, Santa Clara, CA, USA) and Qubit Fluorometer (Invitrogen). The reference human genome version is hg38. The sequencing quality were assessed with the quality assessment software FastQC (v0.11.5) and then low-quality reads were removed using NGSQC (v2.3.3) [16, 17]. The paired-end clean reads were then mapped to the reference genome using HISAT2 (v2.1.0) with default parameters [18]. Gene expression levels were calculated using StringTie (v1.3.3b) [19]. The differentially expressed genes (DEGs) between samples were evaluated by DESeq (v1.28.0) [20]. Thousands of independent statistical hypothesis testing was conducted on DEGs, separately. FDR-corrected $P$-value (FDR $P$ ) of 0.05 was used as a cutoff for significance. Parameters for defining significantly DEGs are $\geq 2$-fold change $\left(\left|\log _{2} F C\right| \geq 1, F C\right.$ : the fold change of expressions) in the transcript data and $P \leq 0.05$. The DEGs were annotated based on the information acquired from the database of ENSEMBL, NCBI, Uniprot, GO, and KEGG. The biological processes analysis of DEGs were identified using Ingenuity Pathway Analysis (IPA) Software (QIAGEN Inc., https://www.qiagenbioinformatics.com/products/ingenuity-pathway-analysis). Volcano plots were generated with the "pheatmap" and "ggplot2" R packages.

\section{Immunohistochemistry (IHC) analysis}

The paraffin-embedded NPC tissues sections were analyzed through IHC using anti-human PNCK (Abcam, Cat \#ab235093). HRP-linked secondary antibodies (Abcam, Cat \#ab205718, UK) were then used followed by DAB treatment. Stained cells were blindly scored based on the staining intensity (negative: 0 ; weak: 1 ; moderate: 2 ; and strong: 3 ) and the abundance of positive cells ( $0 \%: 0 ; 0-5 \%: 1 ; 6-25 \%: 2 ; 26-50 \%$ : 3; $51-75 \%: 4$, and $\geq 76 \%: 5$ ) by two senior pathologists. To obtain the final score, the intensity score(0-3) was multiplied by the extent score(0-5) to identify PNCK expression level.

\section{Gene set enrichment analysis}

GSEA was used to compare expression levels between a priori defined set of genes and, high and low PNCK expression groups in the enrichment of MSigDB Collection (c2.cp.kegg and c5.go.bp. v7.2. symbols.gmt). High and low PNCK was then used as a phenotype label and gene set permutations were carried out 1000 times for each analysis. False discovery rate (FDR) and normalized enrichment score (NES) was used to classify GO and KEGG pathways enriched in differential phenotype.

\section{Wound healing assay}

Transfected CNE-2 and SUNE-1 cells $\left(\sim 1 \times 10^{5}\right.$ cells/well) were seeded in six-well plates and cultured until they reached $80 \%$ confluence. Then the monolayers were wounded by dragging a $1 \mathrm{~mL}$ pipette tip across the cells and then cells were washed to remove cell debris and allowed to migrate for $48 \mathrm{~h}$. Cells were monitored at 0 and $48 \mathrm{~h}$ following wounding and images of wound healing were captured using a DMI 6000 inverted microscope. Cell migration was quantified by measuring the wound healing index; i.e., the 
wound area healed by the cells at $48 \mathrm{~h}$ after scratching relative to the wound area at $0 \mathrm{~h}$, using Image $\mathrm{J}$ software.

\section{Transwell assay}

Transfected cells were digested and resuspended in serum-free RPMI 1640, after which $200 \mu$ of the cell suspension was seeded $\left(1 \times 10^{6} \mathrm{cells} / \mathrm{ml}\right)$ into the upper chambers of an $8 \mu \mathrm{m}$ Transwell filter (Millipore), that were precoated with 1:3 diluted Matrigel (BD Biosciences). RPMI 1640 including with $10 \%$ FBS (700 $\mu \mathrm{l})$ was added to the lower chamber. Subsequently, the plate was incubated for $24 \mathrm{~h}$ at $37^{\circ} \mathrm{C}$ in $5 \% \mathrm{CO}_{2}$. The migration and invasive capability of cells was assessed based on the penetration of the membrane and gel membrane of the matrix, respectively. Following $24 \mathrm{~h}$ incubation, the residual cells in the upper chamber were removed with cotton swabs and dried. Then, migrated and invaded cells were fixed with $4 \%$ paraformaldehyde for $15 \mathrm{~min}$ at room temperature, washed with PBS and stained with $0.1 \%$ crystal violet for $10 \mathrm{~min}$ at room temperature. Cell counting was performed in at least 5 randomly selected fields of view under a DMI 6000 inverted microscope $(\times 200)$.

\section{Experimental metastasis assay in vivo}

Male BALB/c(nu/nu) nude mice (4-6 weeks of age) were purchased from Wushi Experimental Animal Supply Co., Ltd. (Fuzhou, China). Metastasis of PNCK-overexpressing SUNE-1 cells and wild-type cells was given by tail vein intravenous injection. The experiment was terminated 28 days after tumor cell implantation. Then lungs were embedded in paraffin and H\&E staining was performed after euthanasia. Metastatic nodules in the lungs of mice were counted respectively.

\section{Zebrafish and microinjection}

For overexpression of PNCK, the one-cell-stage embryos of Tg (fli1a: EGFP) transgenic line with vascular fluorescently labeled was microinjected with CMV-derived human PNCK gene overexpression vector CMVhPNCK (about 200-300 pg/embryo) using a microinjection apparatus (Eppendorf, FemtoJet 4i). For knock-down of PNCK, RNA targeted editing technology conducted by CRISPR-RfxCas13d (CasRx) system was used [21], in which the endogenous PNCK gene of zebrafish is knocked down by co-injected with 2-3 $\mathrm{nl}$ CasRx capped mRNA $(200 \mathrm{ng} / \mu \mathrm{l})$ and gRNA $(100 \mathrm{ng} / \mu \mathrm{l})$. After 48 hours of injection, the overall fluorescence signal of zebrafish is collected by a stereoscopic fluorescence microscope (SMZ800N). The ImageJ software was used to evaluate the formation of vascular structure and length of intersegmental vessels (ISVs). Moreover, the anti-angiogenic or angiogenesis activity was identified by the basis of the total inhibition of ISVs growth and incomplete sprouting of the ISVs from the dorsal aorta (DA) to the dorsal longitudinal anastomatic vessel (DLAV).

\section{Statistical analysis}

SPSS 17.0 and R software (Version 3.4.4) were performed for all statistical analyses. The quantitative data from at least three independent experiments were shown as mean \pm SD. Student's $t$ test was used to 
compare the differences between groups. Spearman's rho test was made to analyse the correlation among qualitative parameters. All data were determined substantial difference if $P<0.05$.

\section{Results}

\section{Upregulated PNCK in nasopharyngeal carcinoma metastatic tissue}

mRNA expression profiles were performed by mRNA sequencing using 5 NPC metastatic and 5 nonmetastatic tissue to explore the role of PNCK in NPC metastasis. We found 520 upregulated and 808 downregulated mRNAs with the criteria of the threshold value of $P<0.05$ and $\| \log _{2} F C \mid>1.0$ (Fig. 1a). We found that the mean level of PNCK expressed in metastatic samples was twice as high as in nonmetastatic controls (Fig. 1b). The independent cohort GEO datasets (GSE102349) verified the expression level of PNCK was significant higher in advanced (stage III-IV) NPC patients than in patients with early stage (stage I-II) $(P<0.05$, Fig. 1c). Moreover, patients showing high expression of PNCK were significantly associated with worse PFS that indicated poor prognosis(Fig. 1d). Additionally, the protein levels of PNCK were further detected to evaluate its clinical significance in NPC patients using 132 paraffin-embedded NPC tissues by immunohistochemical staining (Fig. 1e). Overall, it showed higher protein levels of PNCK in patients with metastasis than that in the non-metastatic ones $(P<0.05$, Fig. 1f).

\section{Gene Set Enrichment Analysis of PNCK}

Subsequently, we performed genome-wide expression analysis to determine the DEGs in CNE-2 cell lines with PNCK knockdown [11]. Pathway analysis revealed NF-KB and VEGF significant changes of signaling pathway using Ingenuity Pathway Analysis (IPA) (Fig. 2a). Likewise, GSEA was performed to identify GO and signaling pathways in low and high PNCK expression group using 5 NPC metastatic and 5 nonmetastatic tissue by microarray. As shown in Fig. 2b, negative regulation of NF-KB transcription factor activity were enriched in the PNCK low expression phenotype. Although VEGF pathway was not significantly enriched in the high PNCK group, the expression of PNCK was positively associated with VEGFA shown in Fig. $2 \mathrm{c}(r=0.72, P=0.024)$. GEO datasets verified the significant relationship between PNCK and VEGFA ( $r=0.4, P<0.001$, Fig. 2 d). Collectively, these findings revealed that depletion of PNCK may inhibits NF-KB/VEGF signaling pathway in NPC.

\section{PNCK promote NPC cell metastasis in vitro and in vivo}

We constructed NPC cells with PNCK overexpression (oePNCK cells) to assess the PNCK-mediated metastatic abilities in NPC using CNE-2 and SUNE-1 cell lines. Moreover, it was demonstrated that PNCK silencing inhibited NPC cell migration and invasion. In contrast, PNCK overexpression enhanced the metastatic potential of cells as shown in wound-healing and transwell invasion assays (Fig. 3a-c, $P<$ $0.01)$.

Moreover, we attempted to validate the results in vivo with nude mouse model of lung metastasis. SUNE1 cells transfected with control or PNCK overexpression vector were inoculated into nude mice (6 weeks 
old) with tail vein injection. And then we evaluated the number of metastatic lesions in lungs after 4 weeks. The metastatic foci on the lung surface were more plentiful in the oePNCK group than the oeVec group, as shown in Fig. $4 \mathrm{a}, \mathrm{b}(P<0.001)$. Similarly, the number and volume of micrometastases in lungs of mice injected with SUNE-1 cells with overexpressing PNCK exhibited significantly increased (Fig. 4a). These results suggested that PNCK is essentially important for promoting the metastatic of NPC cells.

\section{PNCK is required for angiogenesis in zebrafish}

Zebrafish, serve as a powerful model system increasingly used for cancer research, such as drug discovery and xenografting of cancer cells [22]. Recent observations suggested the possibility of using zebrafish to explore tumor angiogenesis [23]. Through bioinformatics research, it is found that the PNCK gene of zebrafish has more than $60 \%$ homology with humans (Fig. 4c). Given that VEGF signaling pathway was significantly enriched in the PNCK high expression group, we used RNA targeted editing technology to knock down the endogenous PNCK gene using Tg (fli1a: EGFP) transgenic zebrafish embryos to further verify potential correlation between PNCK expression and angiogenesis (Fig. 4d). When the treated embryos developed to 48 hours post fertilization (hpf), the overall fluorescence signal of zebrafish was collected by the fluorescence microscope. Through calculation and statistical analysis, we found angiogenesis among intersegmental vessels (ISVs) of zebrafish was downregulated compared with control group after the knockdown of PNCK gene (Fig. 4e). Similarly, we injected PNCK overexpression plasmid (CMV-hPNCK) and control one (CMV-mCherry) into Tg (fli1a: EGFP) transgenic zebrafish embryos, which were treated with $0.75 \mu \mathrm{mol} / \mathrm{L}$ sunitinib to inhibit angiogenesis, embryos with high level of PNCK caused upregulated angiogenesis among ISVs during 48 hpf (Fig. 4f).

\section{Discussion}

NPC is a malignancy exhibiting high potential of invasion and metastasis, with distant metastasis as a major factor for treatment failure $[24,25]$. Hence, exploring the molecular mechanism of tumor metastasis is crucial to develop effective treatment strategies for NPC patients. In this study, we have confirmed PNCK is highly expressed in metastatic NPC specimens by mRNA sequencing and IHC. By analyzing the GEO cohort, we further validated that PNCK was highly expressed in advanced NPC patients. We demonstrated that upregulation of PNCK promoted migration and invasion of NPC cells in vitro and developed lung metastasis in vivo. Moreover, zebrafish experiments revealed that PNCK overexpression promoted tumor angiogenesis. Our results also highlighted the important role of NF$\mathrm{KB} / \mathrm{VEGF}$ signaling pathway in NPC metastasis.

PNCK located on chromosome Xq28, as a member of CaM kinase, with the enzymatic activity of serine/threonine kinases [10]. It involved in various biological functions including the processes of transcriptional regulation, cell-cycle control, and neurotransmitter release $[8,9]$. Some previous studies have been done to explore the role of PNCK in cancers $[11,13,26]$. In hepatocellular carcinoma, PNCK was highly expressed in tumor samples comparing with non-tumor ones at mRNA and protein levels, and related with a poor prognosis, higher Edmondson grade, higher AFP levels, microvascular invasion and 
intrahepatic metastasis [26]. Additionally, high PNCK expression indicated poor prognosis of clear cell renal cell carcinoma, and associated with advanced T, $\mathrm{N}$ stage and poor differentiation [13]. Previously we demonstrated that NPC tumor tissues present increased PNCK expression, and it regulates cell proliferation and apoptosis with the support of PI3K/AKT/mTOR signaling pathway [11]. However, the underlying mechanism and clinical value of PNCK in tumor metastasis have not been elucidated.

Invasion and metastasis are the major contributors of most treatment failure of cancers [27]. Distant metastasis is responsible for limiting further improvements in NPC treatment [25]. GSEA and IPA analysis were used to elucidate the potential role of PNCK in NPC metastasis via comparing with PNCK-depleted cells. It revealed that the PNCK high expression phenotype enriched in NF-KB/VEGF signaling pathway. Oxygen and other nutrients from vasculature are required to metastases [28]. Previous studies indicated the increased cytosolic $\mathrm{Ca}^{2+}$ activates PNCK, which phosphorylates $\mathrm{IKBa}$ at Ser32 and further triggers $\mathrm{Ca}^{2+}$-dependent NF-KB signaling activity under hypoxia condition [29]. The activated $\mathrm{Ca}^{2+}$-induced NF-KB pathway promotes tumor progression via increasing the transcription of VEGF, thus promoting angiogenesis and cancer development [29]. We used Zebrafish to further explore the potential role of PNCK-mediated angiogenesis in NPC. Interestingly, we found that increased PNCK expression promoted the outgrowth of ISVs of zebrafish in this study, indicating positively regulating NPC angiogenesis. PNCK negatively regulates the ability of NF-KB/VEGF signaling pathway to inhibit tumor invasion and metastasis, providing a novel target for anti-tumor treatment in NPC.

Nevertheless, there are several limitations in this study. Firstly, there were a small sample size of sequencing in our cohort, but we verified from protein level using more tissue samples. Moreover, we lack the complete clinical data which limiting us to evaluate its association with PNCK expression and clinical factors like age, gender, stage, or overall survival. Furthermore, we found that NF-KB/VEGF signaling pathway plays a critical role in NPC metastasis, but the detailed mechanisms regulated by PNCK and corresponding upstream or downstream molecular remains unclear. Therefore, further researches need to be done to explore the mechanisms underlying the interactions between PNCK and NF-KB/VEGF axis in NPC metastasis.

\section{Conclusions}

In conclusion, our study demonstrated that PNCK plays a critical role to mediate NPC metastasis. High expression of PNCK may promote the metastasis and angiogenesis of NPC. It provides new insights that PNCK may be useful to predict future metastasis and as an efficient antiangiogenic target for NPC treatment.

\section{Abbreviations}

NPC: nasopharyngeal carcinom; PNCK: pregnancy upregulated non-ubiquitous calmodulin kinase; VEGF: vascular endothelial growth factor; HER-2: human epidermalgrowth factor receptor-2; GEO: Gene Expression Omnibus; DEGs: differentially expressed genes; IPA: Ingenuity Pathway Analysis; GO: Gene 
Ontology; KEGG: Kyoto Encyclopedia of Genes and Genomes; ISV: intersegmental vessel; DLAV: dorsal longitudinal anastomatic vessel; PFS: progression-free survival.

\section{Declarations}

\section{Ethics approval and consent to participate}

The study was reviewed and authorized by institutional review board of Fujian Medical University Cancer Hospital.

\section{Consent for publication}

Not applicable.

\section{Availability of data and materials}

The public dataset used in this study is freely available at http://www.ncbi.nlm.nih.gov/geo.

\section{Competing interests}

The authors declare that they have no competing interests.

\section{Funding}

The project was funded by Joint Funds for the innovation of science and Technology, Fujian province (2018Y9105); United Fujian Provincial Health and Education Project for Tackling the Key Research, China (2019-WJ-03); Science and Technology Program of Fujian Province, China (2018Y2003); National Natural Science Foundation of China (11974077); and National Natural Science Foundation of China (82072986).

\section{Authors' s contributions}

Sufang Qiu and wanzun Lin conceived the project and designed this study. Xiaochuan Chen analyzed the data and wrote the manuscript. Ying Li and Zongwei Huang helped to performed the experiments. Wenquan Hong helped to perform immunohistochemical analysis. Wankai Fu and Xiandong Lin directed the research. Youliang Weng helped to revise the manuscript. All authors approved the final version for submission.

\section{Acknowledgements}

We thank Dr. Wang and Dr. Liu for useful suggestions.

\section{References}


1. Chen YP, Chan ATC, Le QT, Blanchard P, Sun Y, Ma J. Nasopharyngeal carcinoma. Lancet. 2019;394:64-80.

2. Feng RM, Zong YN, Cao SM, Xu RH. Current cancer situation in China: good or bad news from the 2018 Global Cancer Statistics? Cancer Commun. 2019;39:22.

3. Li YQ, Tian YM, Tan SH, Liu MZ, Kusumawidjaja G, Ong EHW, Zhao C, Tan TWK, Fong KW, Sommat K, Soong YL, Wee JTS, Han F, Chua MLK. Prognostic model for stratification of radioresistant nasopharynx carcinoma to curative salvage radiotherapy. J Clin Oncol. 2018;36:891-9.

4. Li ZL, Ye SB, OuYang LY, Zhang H, Chen YS, He J, Chen QY, Qian CN, Zhang XS, Cui J, Zeng YX, Li J. COX-2 promotes metastasis in nasopharyngeal carcinoma by mediating interactions between cancer cells and myeloid-derived suppressor cells. Oncoimmunology. 2015;4:e1044712.

5. Chan KC, Hung EC, Woo JK, Chan PK, Leung SF, Lai FP, Cheng AS, Yeung SW, Chan YW, Tsui TK, Kwok JS, King AD, Chan AT, van Hasselt AC, Lo YM. Early detection of nasopharyngeal carcinoma by plasma Epstein-Barr virus DNA analysis in a surveillance program. Cancer. 2013;119:1838-44.

6. Lam WKJ, Chan JYK. Recent advances in the management of nasopharyngeal carcinoma. F1000Res. 2018;7:F1000 Faculty Rev-1829..

7. Nussinov R, Muratcioglu S, Tsai CJ, Jang H, Gursoy A, Keskin O. The key role of calmodulin in krasdriven adenocarcinomas. Mol Cancer Res. 2015;13:1265-73.

8. Berchtold MW, Villalobo A. The many faces of calmodulin in cell proliferation, programmed cell death, autophagy, and cancer. Biochim Biophys Acta. 2014;1843:398-435.

9. Monaco S, Rusciano MR, Maione AS, Soprano M, Gomathinayagam R, Todd LR, Campiglia P, Salzano S, Pastore L, Leggiero E, Wilkerson DC, Rocco M, Selleri C, laccarino G, Sankar U, Illario M. A novel crosstalk between calcium/calmodulin kinases II and IV regulates cell proliferation in myeloid leukemia cells. Cell Signal. 2015;27:204-14.

10. Gardner HP, Rajan JV, Ha SI, Copeland NG, Gilbert DJ, Jenkins NA, Marquis ST, Chodosh LA. Cloning, characterization, and chromosomal localization of Pnck, a $\mathrm{Ca}(2+) /$ calmodulin-dependent protein kinase. Genomics. 2000;63:279-88.

11. Xu Y, Wang J, Cai S, Chen G, Xiao N, Fu Y, Chen Q, Qiu S. PNCK depletion inhibits proliferation and induces apoptosis of human nasopharyngeal carcinoma cells in vitro and in vivo. J Cancer. 2019;10:6925-32.

12. Gardner HP, Ha SI, Reynolds C, Chodosh LA. The caM kinase, Pnck, is spatially and temporally regulated during murine mammary gland development and may identify an epithelial cell subtype involved in breast cancer. Cancer Res. 2000;60:5571-7.

13. Wu S, Lv Z, Wang Y, Sun L, Jiang Z, Xu C, Zhao J, Sun X, Li X, Hu L, Tang A, Gui Y, Zhou F, Cai Z, Wang $R$. Increased expression of pregnancy up-regulated non-ubiquitous calmodulin kinase is associated with poor prognosis in clear cell renal cell carcinoma. PloS one. 2013;8:e59936.

14. Deb TB, Zuo AH, Barndt RJ, Sengupta S, Jankovic R, Johnson MD. Pnck overexpression in HER-2 gene-amplified breast cancer causes Trastuzumab resistance through a paradoxical PTEN-mediated process. Breast Cancer Res Treat. 2015;150:347-61. 
15. Burnik Papler T, Vrtacnik Bokal E, Maver A, Kopitar AN, Lovrečić L. Transcriptomic analysis and metaanalysis of human granulosa and cumulus cells. PloS one. 2015;10:e0136473.

16. de Sena Brandine G, Smith AD. Falco: high-speed FastQC emulation for quality control of sequencing data. F1000Res. 2019;8:1874.

17. Patel RK, Jain M. NGS QC Toolkit: a toolkit for quality control of next generation sequencing data. PloS one. 2012;7:e30619.

18. Kim D, Paggi JM, Park C, Bennett C, Salzberg SL. Graph-based genome alignment and genotyping with HISAT2 and HISAT-genotype. Nat Biotechnol. 2019;37:907-15.

19. Pertea M, Pertea GM, Antonescu CM, Chang TC, Mendell JT, Salzberg SL. StringTie enables improved reconstruction of a transcriptome from RNA-seq reads. Nat Biotechnol. 2015;33:290-5.

20. Anders S, Huber W. Differential expression analysis for sequence count data. Genome Biol. 2010;11:R106.

21. Kushawah G, Hernandez-Huertas L, Abugattas-Nuñez Del Prado J, Martinez-Morales JR, DeVore ML, Hassan H, Moreno-Sanchez I, Tomas-Gallardo L, Diaz-Moscoso A, Monges DE, Guelfo JR, Theune WC, Brannan EO, Wang W, Corbin TJ, Moran AM, Sánchez Alvarado A, Málaga-Trillo E, Takacs CM, Bazzini AA, Moreno-Mateos MA. CRISPR-Cas13d induces efficient mRNA knockdown in animal embryos. Dev Cell. 2020;54:805-17.e7.

22. Brown HK, Schiavone K, Tazzyman S, Heymann D, Chico TJ. Zebrafish xenograft models of cancer and metastasis for drug discovery. Expert Opin Drug Discov. 2017;12:379-89.

23. Bao L, You B, Shi S, Shan Y, Zhang Q, Yue H, Zhang J, Zhang W, Shi Y, Liu Y, Wang X, Liu D, You Y. Metastasis-associated miR-23a from nasopharyngeal carcinoma-derived exosomes mediates angiogenesis by repressing a novel target gene TSGA10. Oncogene. 2018;37:2873-89.

24. Mao YP, Tang LL, Chen L, Sun Y, Qi ZY, Zhou GQ, Liu LZ, Li L, Lin AH, Ma J. Prognostic factors and failure patterns in non-metastatic nasopharyngeal carcinoma after intensity-modulated radiotherapy. Chin J Cancer. 2016;35:103.

25. Zhang L, Huang Y, Hong S, Yang Y, Yu G, Jia J, Peng P, Wu X, Lin Q, Xi X, Peng J, Xu M, Chen D, Lu X, Wang R, Cao X, Chen X, Lin Z, Xiong J, Lin Q, Xie C, Li Z, Pan J, Li J, Wu S, Lian Y, Yang Q, Zhao C. Gemcitabine plus cisplatin versus fluorouracil plus cisplatin in recurrent or metastatic nasopharyngeal carcinoma: a multicentre, randomised, open-label, phase 3 trial. Lancet. 2016;388:1883-92.

26. Cho YA, Choi S, Park S, Park CK, Ha SY. Expression of pregnancy up-regulated non-ubiquitous calmodulin kinase (PNCK) in hepatocellular carcinoma. Cancer Genomics Proteomics. 2020;17:74755.

27. Oh E, Kim YJ, An H, Sung D, Cho TM, Farrand L, Jang S, Seo JH, Kim JY. Flubendazole elicits antimetastatic effects in triple-negative breast cancer via STAT3 inhibition. Int J Cancer. 2018;143:197893.

28. Niu Y, Bao L, Chen Y, Wang C, Luo M, Zhang B, Zhou M, Wang JE, Fang YV, Kumar A, Xing C, Wang Y, Luo W. HIF2-induced long noncoding RNA RAB11B-AS1 promotes hypoxia-mediated angiogenesis 
and breast cancer metastasis. Cancer Res. 2020;80:964-75.

29. Sang LJ, Ju HQ, Liu GP, Tian T, Ma GL, Lu YX, Liu ZX, Pan RL, Li RH, Piao HL, Marks JR, Yang LJ, Yan Q, Wang W, Shao J, Zhou Y, Zhou T, Lin A. LncRNA CamK-A regulates $\mathrm{Ca}(2+)$-signaling-mediated tumor microenvironment remodeling. Mol Cell. 2018;72:71-83.e7.

\section{Figures}

a

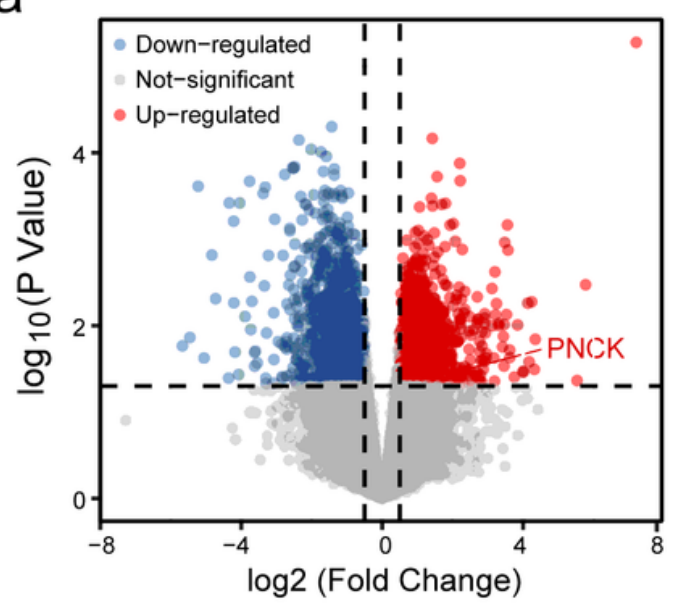

b

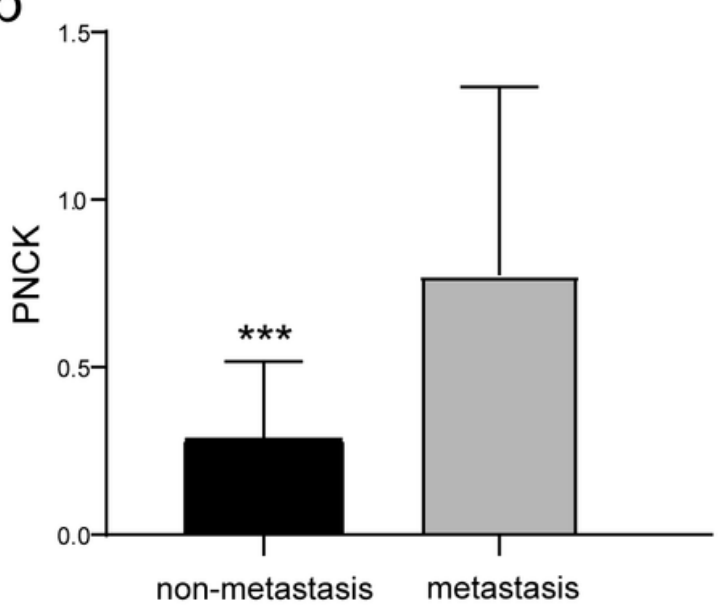

e

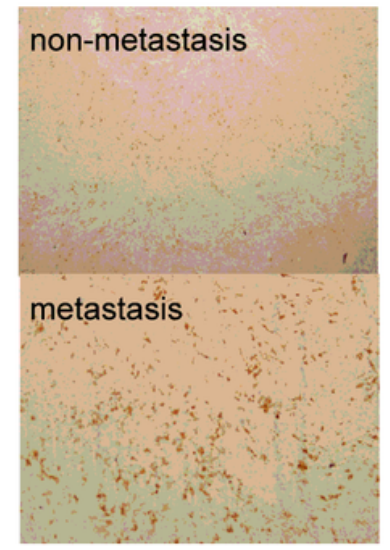

C

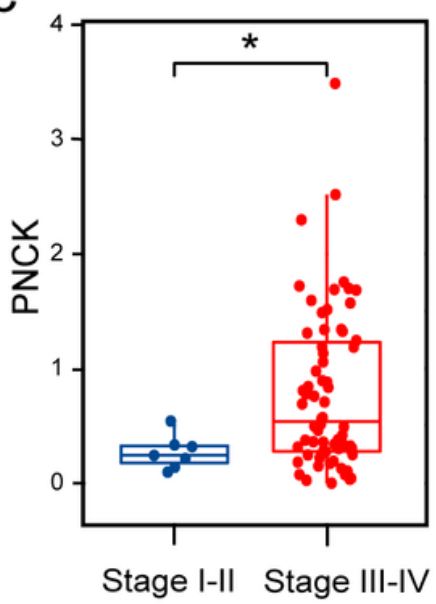

$f$

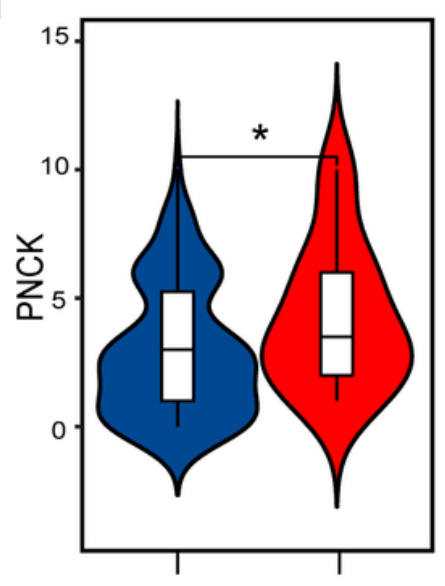

non-metastasis metastasis

\section{Figure 1}

High expression of PNCK in NPC metastatic tissue. a The volcanic plot showing the distribution of significant upregulated or downregulated differentially expressed genes, with PNCK among these upregulated genes, using 5 metastatic and 5 non-metastatic NPC tissue. b Relative expression of PNCK detected by mRNA sequencing. c GSE102349 cohort indicating the higher PNCK expression in advanced NPC patients (stage III-IV) than in patients with early stage (stage I-II). d GSE102349 cohort indicating the higher PNCK expression were associated with worse PFS. e Validation of the protein expression of PNCK 
using paraffin-embedded with NPC non-metastatic and metastatic tissue assessed by immunohistochemistry, and $f$ the quantification of PNCK. Student's t-test, * $P<0.05$.

a

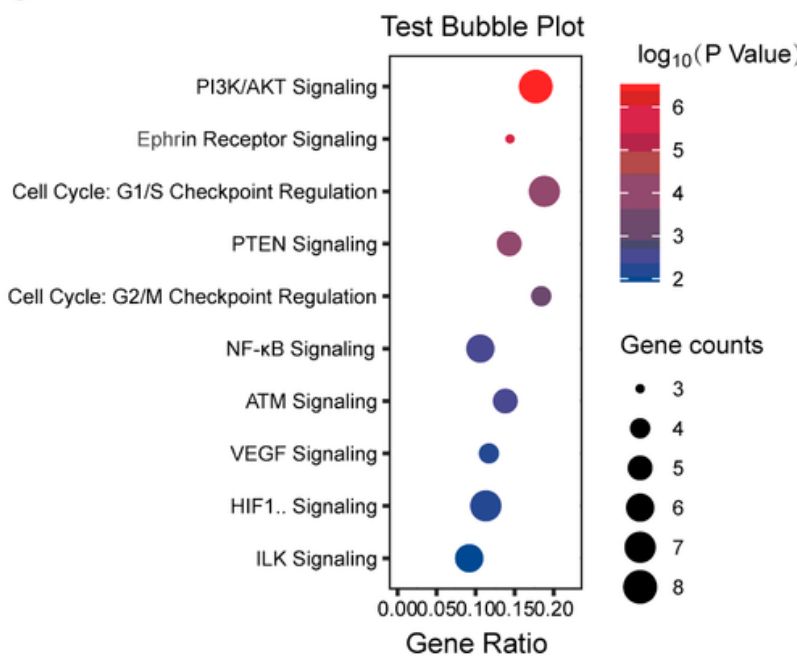

C

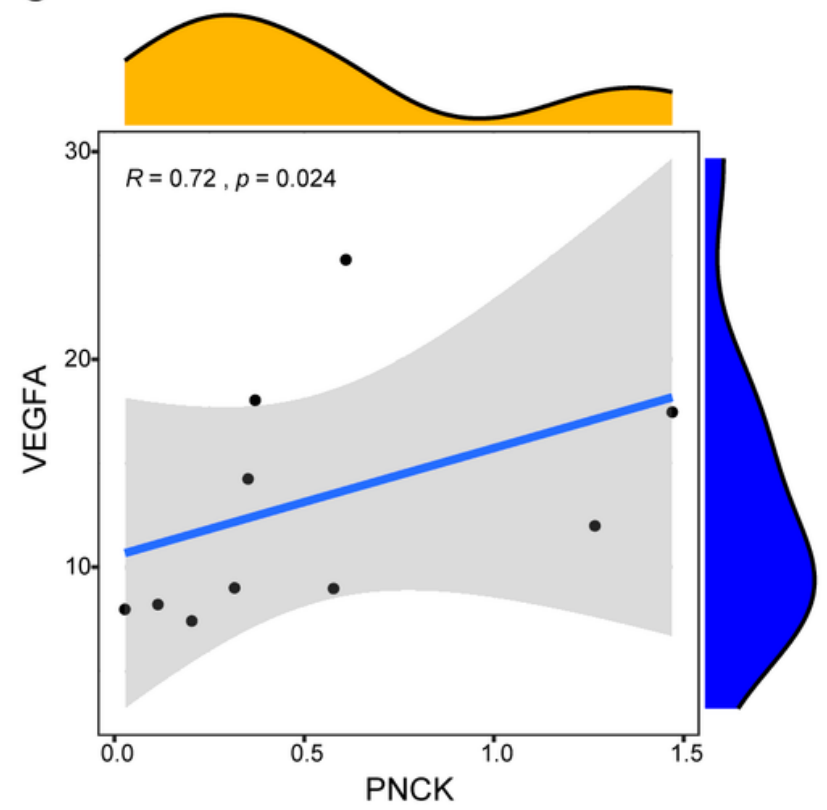

b Enrichment plot:
GO_NEGATIVE_REGULATION_OF_NF_KAPPAB_TRANS CRIPTION_FACTOR_ACTIVITY

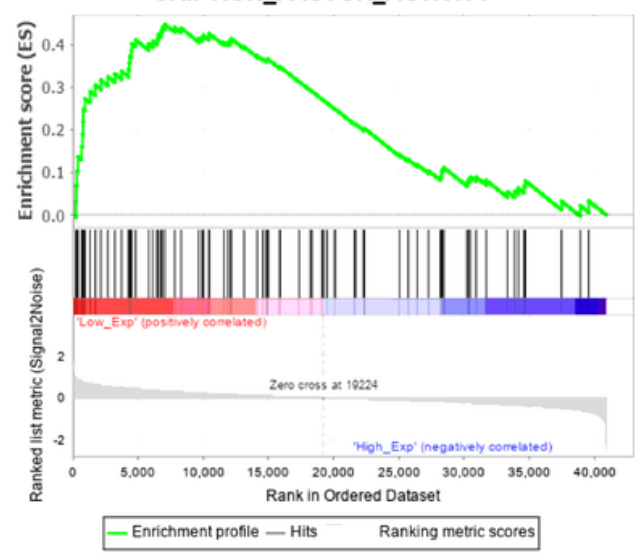

d

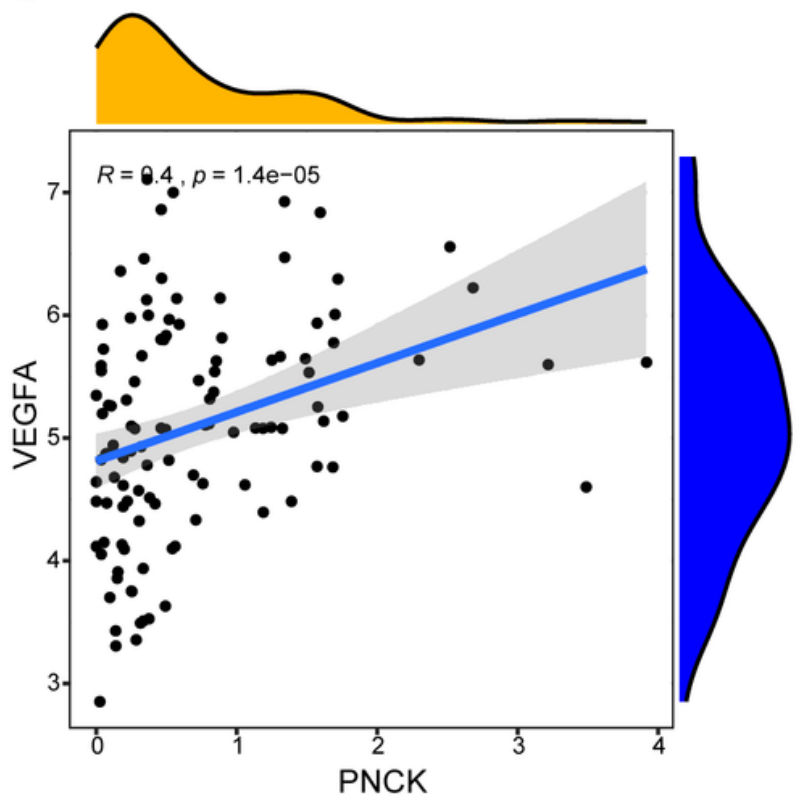

Figure 2

Gene functional enrichment analyses. a Dot plot of Ingenuity Pathway Analysis (IPA) revealed NF-KB and VEGF significant changes of signaling pathway. b GSEA showed negative regulation of NF-KB transcription factor activity enriched in the PNCK low expression phenotype. The correlation between PNCK and VEGFA in c our cohort and d GSE102349 cohort. 
a
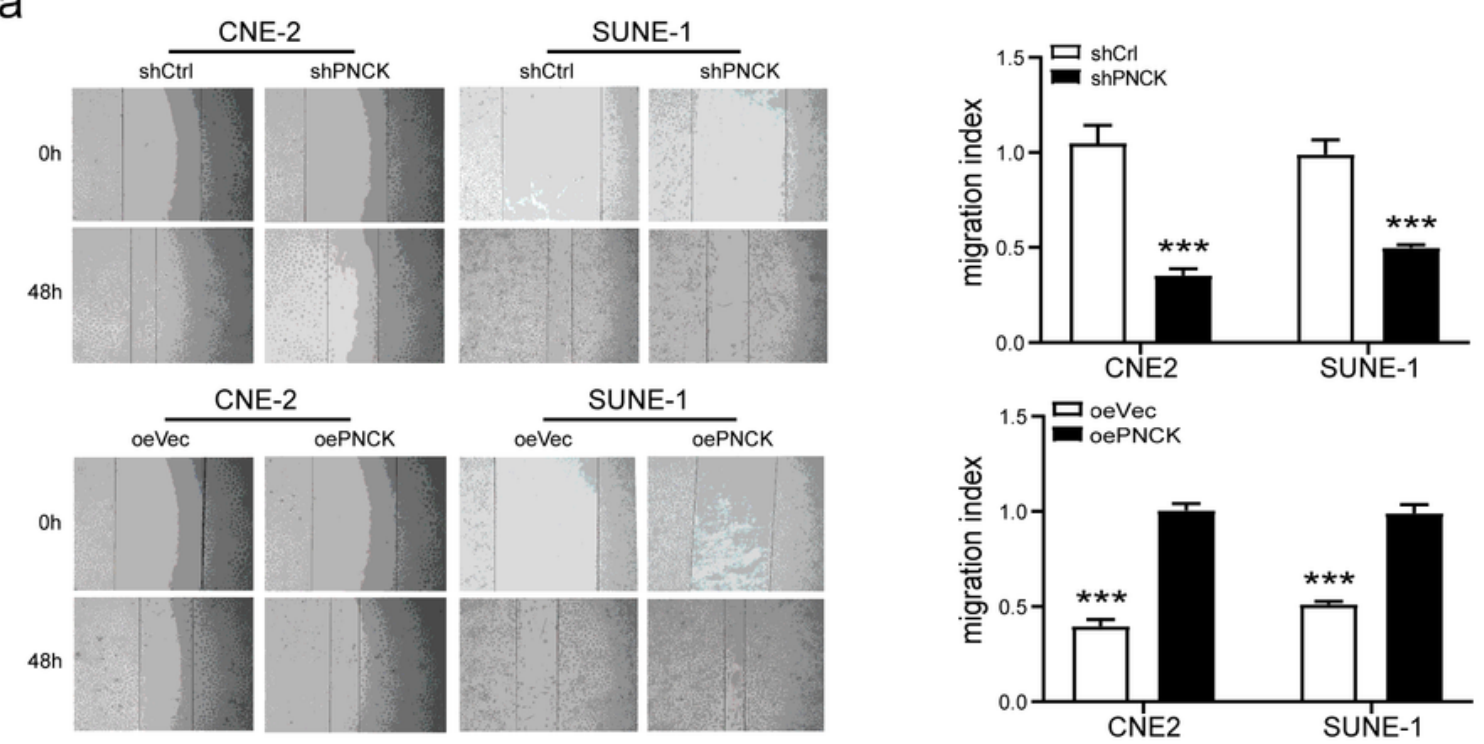

b
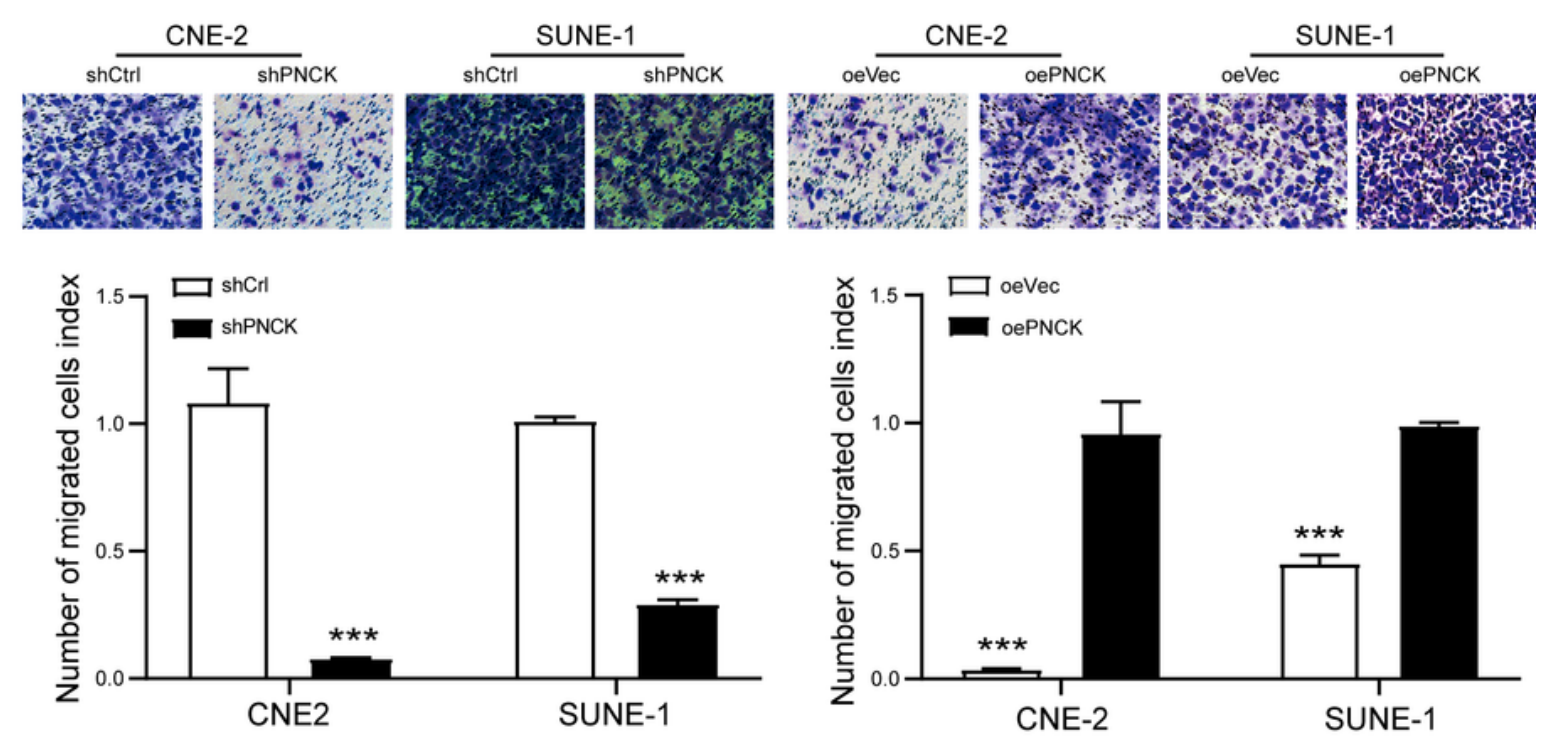

C
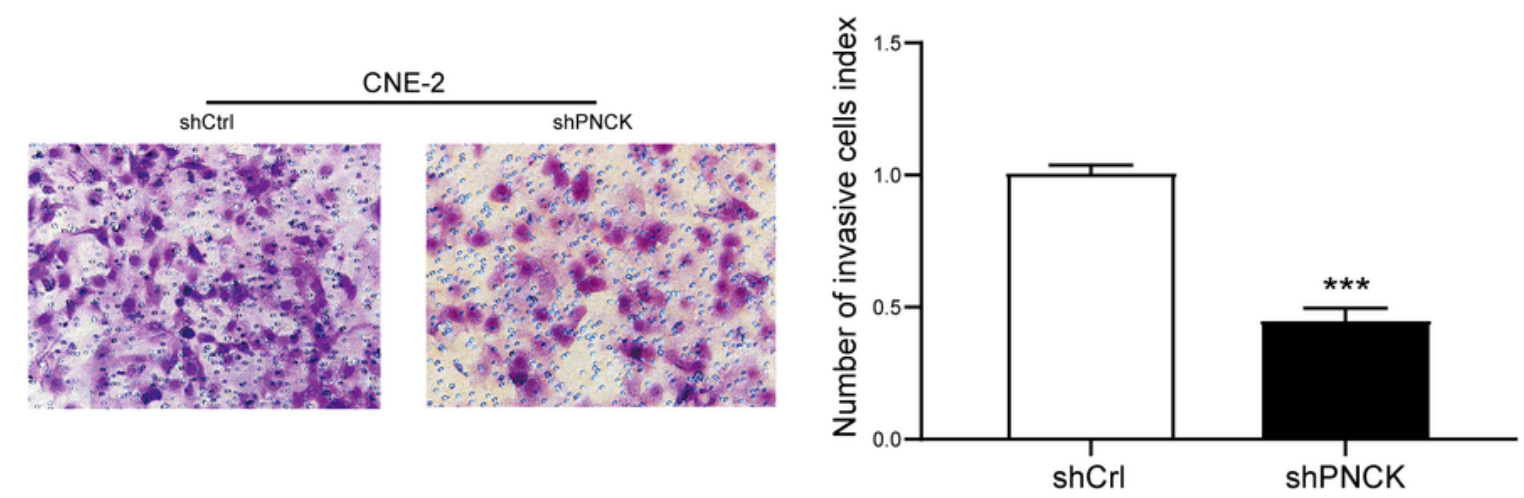

Figure 3

Representative results and quantification of in vitro experiment for constructed NPC cells. a PNCK knockdown (shPNCK) inhibited NPC cells migration distance, while overexpression of PNCK promoted cells migration as showed by wound healing assays. b, c PNCK overexpression (oePNCK) enhanced the invasion and migrated potential of NPC cells as assessed by Transwell assays. Student's t-test, ** $P<$ 0.01 , *** $P<0.001$. 
a
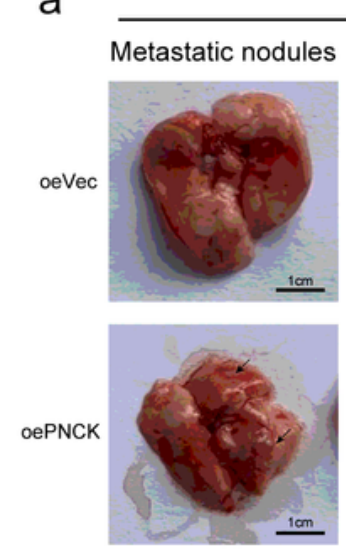

C human
zebrafish

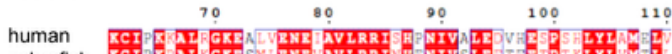

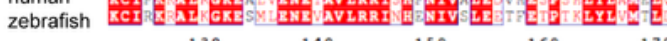

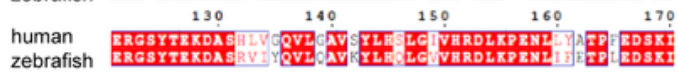

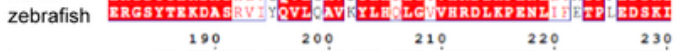

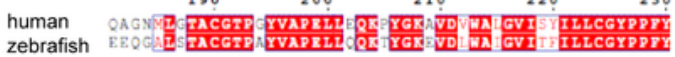

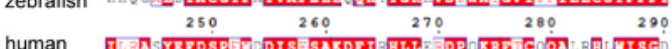

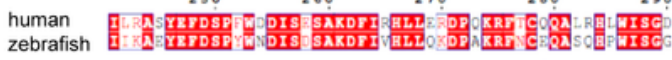

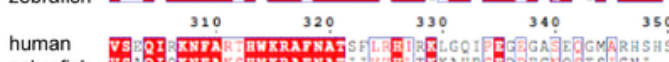
e
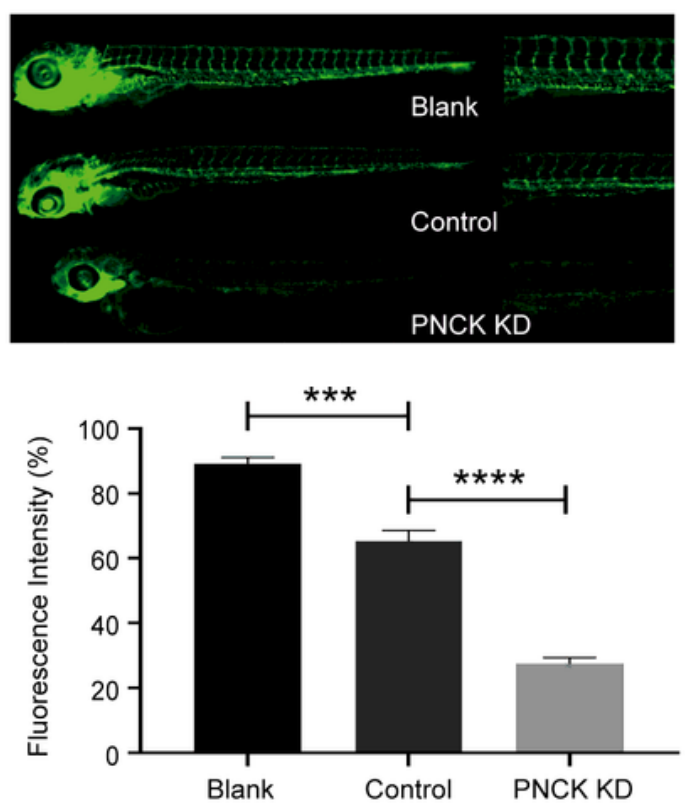

b

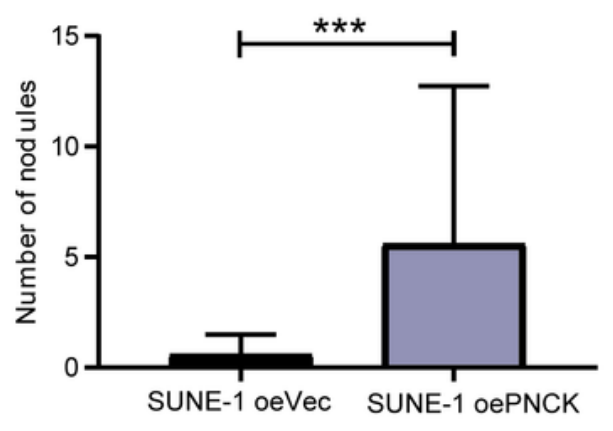

d
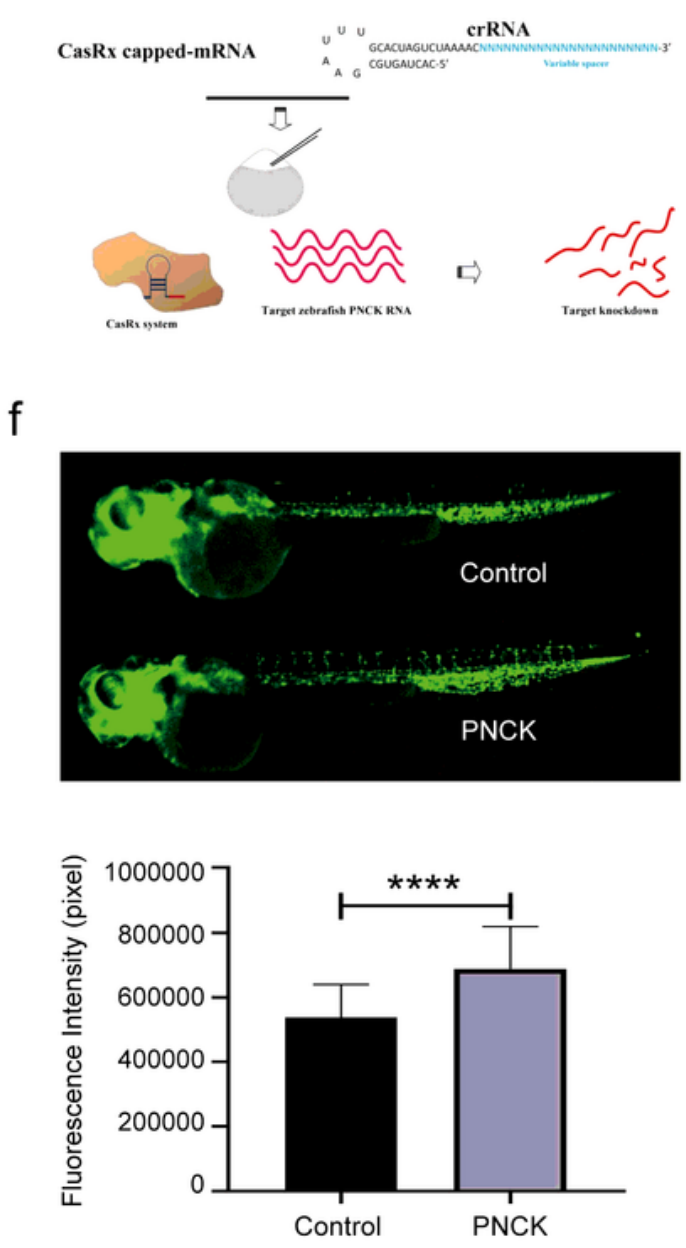

\section{Figure 4}

PNCK promotes lung metastasis and angiogenesis in NPC in vivo. a Representative images and b quantification of lungs metastatic foci of mice after injecting SUNE-1 cells constructed with overexpression of PNCK or control vector via tail vein injection. Arrows indicate surface metastatic nodules. $c$ The aligned results of PNCK amino acid sequence between human and zebrafish. $d$ The schematic diagram of RNA cutting technology in zebrafish embryo. e Fluorescence intensity of 
intersegmental vessels in Tg(fli1a:EGFP) embryos inoculated with blank, control or PNCK KD vector. $f$ Fluorescence intensity of intersegmental vessels in $\mathrm{Tg}$ (fli1a:EGFP) embryos inoculated with control or PNCK vector. Student's t-test, ${ }^{\star \star \star} \mathrm{P}<0.001$. 